\title{
Passive voice should be avoided - or should it?
}

JULIE MEYER INGEMANSSON \& TORBEN JUEL JENSEN

Numerous language campaigns in Denmark have tried to regulate the written language use of public employees, and the focus has generally been on certain pieces of 'good advice', such as 'avoid passives'. However, the effect of these recommendations on the receivers of administrative texts produced by public employees has never been assessed empirically in any satisfactory way.

This article presents a method to investigate the effect (or lack thereof) of passives on readers' comprehension of and attitudes towards letters from the public sector. In a between-subject design, two groups of 10 participants read the same letters in two versions: a version with many passives and a version without passives. The results suggest that passives have no effect on the degree of comprehension of the letters, and only a limited effect on the readers' attitudes towards the text, with perceived 'personalness' as an exception. The results also show that people without higher education have a lower degree of comprehension of the letters than people who have attended higher education.

The article is concluded by an assessment of the experimental design and a discussion of factors that might influence the results, such as the type of passive used, the context and subject matter of the texts. We argue that the method is valid and can be used to establish an empirical basis from which it is possible to provide advice on language use that takes the receiver's perspective into consideration. In particular, we argue that the recommendation commonly found in language campaigns that passives should be avoided is not a particularly relevant piece of advice, or at least one that needs to be presented in a considerably more nuanced way than it usually is. 